Itinéraires Itinéraires

Littérature, textes, cultures

2009-2 | 2009

Caraïbe et océan Indien

\title{
Les Cahiers de Madeleine ou la vie d'une Mulâtresse de Cayenne
}

\section{Monique Blerald}

\section{(2) OpenEdition}

1 Journals

Édition électronique

URL : http://journals.openedition.org/itineraires/266

DOI : 10.4000/itineraires.266

ISSN : 2427-920X

Éditeur

Pléiade

\section{Édition imprimée}

Date de publication : 1 juillet 2009

Pagination : 65-76

ISBN : 978-2-296-09639-4

ISSN : $2100-1340$

\section{Référence électronique}

Monique Blerald, "Les Cahiers de Madeleine ou la vie d'une Mulâtresse de Cayenne », Itinéraires [En ligne], 2009-2 | 2009, mis en ligne le 02 juin 2014, consulté le 10 décembre 2020. URL : http:// journals.openedition.org/itineraires/266 ; DOI : https://doi.org/10.4000/itineraires.266

Ce document a été généré automatiquement le 10 décembre 2020.

\section{(c)}

Itinéraires est mis à disposition selon les termes de la licence Creative Commons Attribution - Pas d'Utilisation Commerciale - Pas de Modification 4.0 International. 


\title{
Les Cahiers de Madeleine ou la vie d'une Mulâtresse de Cayenne
}

\author{
Monique Blerald
}

Chaque personne est un livre qui s'ignore.

Michel Daniel

1 Francine Château présentait en mars 2008, devant une vaste assemblée à l'auditorium de la mairie de la ville de Rémire-Montjoly en Guyane, les écrits de sa mère : La Vie d'une Mulâtresse de Cayenne. 1901-1997. Les Cahiers de Madeleine. Une lecture attentive de ce récit de vie fait apparaître que cet écrit n'est pas seulement pour la Mulâtresse Madeleine Tichette un outil d'accompagnement qui permet à l'écrivain, hantée par sa propre vie et par le besoin de se dire, de donner du sens à sa vie passée comme présente. Ce texte est aussi un travail d'exploration et de compréhension de la vie des bourgeois créoles de Guyane durant la période coloniale. L'auteur essaie d'y appréhender les valeurs prônées par cette classe sociale à laquelle elle appartient. Aussi, nous tenterons d'analyser dans cet ouvrage, outre la dimension affective présente dans le texte, d'une part, la signification donnée aux événements - personnels et historiques - et, d'autre part, les stratégies d'écriture mises en place par rapport à l'imaginaire et à l'identité créoles.

\section{Le témoignage d'une Cayennaise sur la vie coloniale}

Depuis une décennie, les récits de vie connaissent un regain d'intérêt en Guyane. La revendication des identités régionales peut expliquer ce retour vers le patrimoine et vers ces vies passées, dans lesquelles le lecteur guyanais essaie de se replonger et de se retrouver. Un bon accueil a été réservé à La Vie d'une Mulâtresse de Cayenne. 1901-1997. Les Cahiers de Madeleine, comparativement aux autres récits de vie publiés précédemment par des hommes et des femmes reconnus pourtant pour leur implication dans la vie sociale ou politique guyanaise. Rappelons quelques-uns de ces titres. Le récit de vie intitulé Man Vévé raconte (1994) relate la vie d'une femme impliquée sur le plan associatif et politique dans une commune située sur le littoral, la commune de Mana, encore marquée par 
l'empreinte de sa fondatrice, la Mère Anne-Marie Javouhey. Les ouvrages de Roger Lam Chan (De la Guyane au Rhin et Danube, 1998) et de Claude Vernet (Un enfant de France, 2002) mettent l'accent sur les soldats guyanais ayant fièrement défendu la mère patrie, pendant la Seconde Guerre mondiale et la guerre d'Algérie. L'ouvrage d'Yvette Roblin, Guyane je t'aime (malgré tout), publié en 2004, décrit la vie d'une métropolitaine venue s'installer dans le département, après son divorce, seule avec deux enfants. L'auteur met en scène des situations, des personnages ou des espaces peut-être peu évocateurs pour l'imaginaire créole.

3 En revanche, la réception de La Vie d'une Mulâtresse de Cayenne. Les Cahiers de Madeleine est tout autre. Certes, on pourrait avancer le soutien du monde associatif, l'investissement et le dynamisme de la famille en ce qui concerne la publicité faite autour du livre. Mais nous préférons mettre en avant la dimension affective liée à cet ouvrage. Et, pour reprendre la terminologie du sociologue Daniel Bertaux (2005: 52), nous évoquons «la force expressive » dont est chargé ce texte, qui parle directement à l'imaginaire du lecteur.

La Vie d'une Mulâtresse de Cayenne raconte la vie d'une femme, Madeleine Tichette, épouse Château, née le 11 novembre 1901 à Cayenne, en Guyane, à l'époque colonie française d'Amérique. Le regard porté sur Cayenne n'est pas un regard exogène, mais endogène. Il ne s'agit pas, en effet, de celui d'un chroniqueur, d'un explorateur ou d'un missionnaire européen de passage mais de celui d'une native-natale, d'une Mulâtresse, dont certains membres de la famille résident encore à Cayenne. Le premier ouvrage présentant la ville coloniale à travers le regard d'un fils du pays était Atipa, premier roman publié en créole en 1885, par un certain Alfred Parépou, pseudonyme emprunté par un Blanc créole ou un Mulâtre.

Les trois cahiers contenaient toute l'histoire de sa traversée de ce vingtième siècle, d'une belle écriture sans rature avec parfois des redites; elle s'en apercevait et notait "bis repetita" [...]. Nous avons décidé d'en publier certains extraits qui sont la mémoire de la vie guyanaise à cette époque qui semble déjà si lointaine (Tichette, $2007: 8-9)$,

écrit sa fille Francine dans l'avant-propos, qui guide le lecteur et sert «de discours d'escorte au texte narratif » (Abastado, $1983: 19$ ).

Madeleine Tichette, l'auteur-narrateur, se pose en témoin de sa génération et de son époque. Elle présente la vie guyanaise. Elle examine les préoccupations, les comportements, les valeurs et loisirs des Créoles :

Et la vie continuait, les Guyanais avaient d'autres objectifs. Ils voulaient une maison pour la retraite, les plus riches voulaient une villa au bord de la mer... Ils étaient atteints d'une fringale de voyages. Ils allaient à Paramaribo, capitale de la Guyane hollandaise... Ils allaient aussi au Brésil chez les "Cougnades" comme on les appelait à Cayenne... Les fonctionnaires partaient en congé administratif à Vichy soigner un foie colonial qui avait eu quelques secousses avec le ti-punch glacé, citronné au sirop de cannelle. (Tichette, 2007 : 146)

6 Les mentalités ont-elles vraiment changé ? Les Guyanais, bien au contraire, continuent à voyager ; les principales destinations étant le Surinam, le Brésil, les Antilles, la France. Ils vont également en Afrique ou en Amérique du Nord. Ils continuent à se rendre en France dans les stations thermales pour soigner rhumatismes et allergies.

Précisons que, chez Madeleine Tichette comme chez d'autres Créoles, le terme générique Guyanais désigne plus particulièrement le Créole Guyanais. Les autres communautés culturelles les plus représentatives, à son époque, sur le plan économique, les Chinois ou les Syriens, ne sont pas laissées de côté par l'auteur qui décrit leur désir d'ascension 
sociale. Ce qui nous amène à constater l'esprit d'ouverture de Madeleine Tichette, qui s'intéresse à la diversité ethnique et culturelle caractérisant son pays.

Il arrive que l'auteur-narrateur justifie un comportement personnel en l'assimilant à un comportement collectif. Celui-ci prenant valeur de paradigme devient une caractéristique culturelle, dans laquelle d'ailleurs peuvent se reconnaître certains lecteurs créoles :

Tout était prétexte à la fête. On aimait la bonne chère, les gens riches avaient de bonnes bouteilles dans leurs caves. Le Guyanais est gai par nature; on dansait au son de l'accordéon, de la clarinette et du trombone; on vivait en communauté : le soir on mettait des chaises dehors et allez-y donc, tripotages, ragots, bavardages, au moins on communiquait et dès qu'il y avait un service à rendre, une peine à soulager, tels des boys scouts on disait : "prêt". (Ibid. : 110)

9 Claude Abastado explique à ce sujet que dans ce «moi individuel», le « on social » se retrouve, par des énoncés de type axiomatique ou extensionnel. Ces énoncés que constituent proverbes, préceptes ou maximes attribuent alors une qualité, une manière de penser, une conduite individuelle à tous les membres d'un groupe social ou d'un milieu (Abastado, 1983 : 18).

10 Toutefois, on remarquera que Madeleine Tichette fait allusion à un bal populaire, avec violon, accordéon et clarinette, auquel se rendent surtout ceux qui appartiennent à la même classe sociale qu'elle, c'est-à-dire les Mulâtres. Elle ne mentionne à aucun moment dans son ouvrage l'existence des bals populaires régalant le peuple au son du tibwa et surtout du tambour, instrument symbolique rappelant la terre des ancêtres africains dans la revendication nègre. Sa curiosité intellectuelle et son esprit d'ouverture la poussent néanmoins à s'intéresser aux traditions populaires créoles, telles que le conte ou le carnaval. Les refrains glissés çà et là dans le texte, les portraits de carnavaliers ou de conteurs populaires traduisent son désir profond de connaitre son pays mais aussi son attachement à celui-ci. Son intérêt pour le créole, même si celui-ci demeure une langue de communication essentielle entre les différents groupes socioculturels en Guyane, montre également que la Mulâtresse cherche à connaître ses origines et semble, contrairement à ceux de sa classe, se réapproprier la part de culture nègre que lui a léguée son aïeule.

11 Tout en respectant soigneusement la chronologie des événements - des historiens (Mam Lam Fouck, 1996) pourraient attester l'exactitude des repères choisis -, Madeleine Tichette propose, néanmoins, une vision très personnelle de l'histoire coloniale. Elle analyse scrupuleusement l'impact du régime de Vichy sur la Guyane en 1944 et le comportement plus ou moins ambigu des Guyanais durant la dissidence :

Et les Guyanais admiraient le courage de De Gaulle. Mais le gouvernement, un peu l'administration et totalement le clergé ne cessaient de louanger le grand vainqueur de Verdun, Pétain, pour son courage, son patriotisme, etc. Il y eut des messes à la cathédrale pour l'éclairer et le soutenir. Les enfants de l'école des Sœurs circulaient dans les rues en braillant :

"Maréchal, nous voilà, Devant toi, le sauveur de France."

Quand on apprit que notre grand compatriote Félix Eboué, administrateur des Colonies, s'était rallié à De Gaulle, les Guyanais furent électrisés et lui envoyèrent un message pour lui apprendre notre ralliement... La situation était confuse. (Tichette, $2007:$ 192)

12 Madeleine Tichette s'intéresse à la révolte des tirailleurs sénégalais envoyés en Guyane en 1946 pour rétablir l'ordre après les émeutes suscitées par la mort du député périgourdin Jean Galmot. Elle souligne la présence des forçats en Guyane et met l'accent sur l'accord 
passé entre le pouvoir colonial local et l'administration pénitentiaire. Celle-ci autorisait les bagnards qui avaient accompli leur peine à résider dans la colonie. Ils devenaient alors employés de maison chez les bourgeois créoles ou étaient réquisitionnés pour les corvées d'intérêt général (Épailly, 1995):

Des grands chariots tirés par des buffles, dans un désordre infernal, passaient dans les rues pour le chargement et le déchargement des "tinettes". Les forçats étaient chargés de ce travail répugnant, nauséabond, éclairés par des "Lampes Diez" pour pénétrer dans les cours sombres à quatre heures du matin, heure de leur passage. (Tichette, 2007 : 55)

13 Cependant, on retiendra dans ces évocations historiques la description anecdotique de faits et de personnages auxquels elle accorde une valeur souvent sentimentale. Ainsi, lorsqu'elle fait allusion à Collot d'Herbois, prêtre réfractaire envoyé sous la Révolution française en Guyane, elle le rattache aux ancêtres de ses amies, qui l'avaient connu durant sa déportation. Il en est de même d'Hippolyte, le forçat martiniquais que sa mère Eugénie Luherre avait embauché pour entretenir le petit jardin familial, ou encore des frères Pierre et Paul Colin, anciens déportés devenus cochers de ses amis, les époux Doria.

Pourtant, elle ne cite ni Dreyfus, ni Denis Seznec, les célèbres résidents des îles du Salut, ni le combat d'Albert Londres qui a permis la fermeture définitive du bagne en Guyane en 1953. Elle passe par ailleurs sous silence un moment clé de l'histoire de la Guyane. Il s'agit de la fin de la colonisation et de l'accès à un nouveau statut : la départementalisation. Pourquoi ce vide ? Est-ce parce que cette période correspondait avec la disparition de son frère Édouard, dernier parent encore en vie, et avec les tracasseries administratives, juridiques qui lui furent liées? Est-ce à cause des préparatifs mais surtout des interrogations que suscitaient à cette époque son départ et celui de sa famille pour la France ? Son mari, parti à la retraite, souhaitait en effet un changement de cadre de vie et un maximum d'instruction pour leurs filles. Elle allait quitter sa chère Guyane, sa mère et ses amis, et voguer vers l'inconnu :

J'étais désemparée; des pour et des contre se baladaient dans ma tête... Fallait-il quitter tout cela pour l'inconnu, le risque et y conduire mes pauvres petites innocentes? (Ibid. : 204)

Quoi qu'il en soit, Madeleine Tichette a le mérite de répertorier les grandes phases de l'histoire de la Guyane. L'importance et le rôle de la mémoire sont ici significatifs. La mémoire reconstruit le passé, transforme le réel vécu, opère des choix mais elle peut aussi laisser de côté des pans de l'histoire individuelle ou collective. En ce sens, l'oubli peut être durable et bénéfique. Se perdre soi-même, c'est-à-dire oublier, peut être un moyen de se protéger, de résister aux épreuves, précise Joël Candau (1998 : 124). N’est-ce pas une attitude souvent adoptée par Madeleine Tichette?

\section{Quête identitaire, quête mémorielle}

La Vie d'une Mulâtresse de Cayenne se présente surtout comme " un récit rétrospectif » que Madeleine «fait de sa propre existence » (Lejeune, 1996: 14), à la demande de ses trois filles Jeanne, Josée et Francine. D'abord enfants, elles «l'écoutent blotties à ses pieds, dans un silence respectueux, raconter l'histoire de sa vie par fragments » (Tichette, 2007 : 7), puis plus tard adultes, loin d'elle dans leurs foyers respectifs, elles lui demandent d'écrire tout simplement son histoire. Ce qu'elle s'emploie à faire à partir de 1989, à la 
naissance de sa première arrière-petite-fille Marie, tout en interdisant formellement qu'on lise les trois cahiers avant sa mort, à Paris, en 1997.

Dans ces cahiers qui retracent sa vie de 1901, date de sa naissance, à 1991 - date où elle décide de s'arrêter d'écrire pour des raisons de santé -, l'auteur-narrateur raconte ses souvenirs d'enfance et d'adolescence, sa vie de jeune épouse et de jeune mère en Guyane, puis elle fait état de son expérience métropolitaine à Paris. Ces écrits constituent le corps de l'ouvrage que les filles de Madeleine Tichette décident de publier en 2007. Si l'on se réfère à la définition de Daniel Bertaux concernant le récit de vie, $L a$ Vie d'une Mulâtresse de Cayenne peut être considéré comme tel. Nous avons bien « une description sous forme narrative d'un fragment d'une expérience vécue [...]. Un sujet raconte à une autre personne, chercheur ou pas, un épisode quelconque de [cette] expérience " (Bertaux, 2005 : 36).

L'auteur-narrateur, en effet, s'adresse tout d'abord à ses filles et à ses petits-enfants, des interlocuteurs en vie. La dédicace placée dans la deuxième partie de l'ouvrage est explicite: «Pour mes filles que j'aime, mes petits-enfants, mes arrière-petits-enfants, Marie, Lucas. Toute ma tendresse. » (Tichette, $2007: 205)$.

Dans la dernière partie de ses cahiers, elle s'adresse également à un être cher disparu, Edgard Château, son mari pendant trente ans. Cependant, au-delà de l'acte d'amour, il y a surtout le sentiment du devoir accompli, qui revient comme un leitmotiv dans le récit :

Je revis notre vie ensemble avec ses jours heureux et ses jours malheureux comme dans toute vie. Nos enfants ont grandi. Je crois que j'ai bien rempli ma tâche comme tu le voulais et que tu es satisfait de moi. Elles ont de l'instruction, une situation confortable, de belles demeures, des maris gentils et nous nous aimons bien. Elles sont belles, coquettes, prévenantes et attentionnées pour leur maman. (Ibid. : 277)

C'est une épouse, une mère comblée, qui se retire paisiblement :

La nuit approche ; la mort arrive à grands pas. Il est temps de clore mon livre..., ce livre dans lequel j'ai mis tout mon cœur, toutes mes pensées, franchement. J'ai eu beaucoup de chance dans ma vie, une enfance heureuse, comblée par une mère chérie, un mari en or. J'ai voulu des enfants et je n'ai pas été déçue. Elles m'ont donné de grandes satisfactions et m'entourent encore de tendresse et de prévenances. (Ibid. : 280)

Madeleine peut donc être perçue comme une héroïne ordinaire, derrière laquelle se cache un esprit sensible.

Pas clairement décrite, aux contours imprécis, cette Mulâtresse est à la recherche de ses origines ; c'est-à-dire en quête de son identité. Elle découvre que son aïeule "Cici » était une jeune Négresse achetée un jour de vente aux esclaves sur la Place du Marché de Rémire. Elle est abandonnée par un père qu'elle n'a pas connu. Très jeune, elle perd sa mère Anna, mais trouve en sa marraine, Eugénie Lhuerre, une Créole blanche née en 1861, une seconde mère. Scolarisée chez les sœurs de Saint-Joseph de Cluny, elle obtient son «brevet simple». Elle est par la suite employée au Trésor où elle rencontre Edgard Château, son mari et le père de ses trois filles. Derrière une apparente réussite qui semble faire fi des débuts difficiles, se cache une profonde angoisse existentielle, que le texte narratif met à nu. La description minutieuse de la maison familiale et du petit jardin situés au 59 de la rue Lalouette, des sites fréquentés pendant la jeunesse, la multiplicité des personnages, le dédoublement des êtres et des événements, créent une sensation de confusion et de vertige et peuvent être perçus comme des mécanismes de défense obsessionnels, nécessaires à la reconstruction personnelle, suite au drame intérieur que 
l'auteur-narrateur a vécu. À travers les portraits de proches, d'amis en proie à toutes les vicissitudes de l'existence, et dont la vie est une lutte, tels son vieil oncle Édouard ou encore ses amies d'enfance, l'auteur-narrateur nous conduit tout simplement jusqu'à elle.

Certains thèmes apparaissant régulièrement dans les récits de vie et correspondant à l'attente des lecteurs : naissance, portrait de famille, petite enfance, baptême, première communion, premier amour, accident ou maladie sont aussi présents dans La Vie d'une Mulâtresse de Cayenne. Ces stéréotypes thématiques nous aident à découvrir dans l'ouvrage les joies et les peines de la Mulâtresse.

À côté donc des instants de bonheur, les moments de souffrance sont aussi passés en revue. La douleur éprouvée lors de la perte d'êtres chers reste toujours intense, qu'il s'agisse de celle de sa sœur :

Jeanne est morte le 9 novembre. Elle avait dix-neuf ans. Le surlendemain, je devenais majeure.

J'avais souffert en perdant ma sœur: c'était un grand vide, ma première peine. J'allais souvent au cimetière lui porter des fleurs. Je devins plus pieuse, la prière me rapprochant d'elle. Mais à mon tour, je dépérissais : je ne pesais plus que quarantedeux kilos. (Ibid. : 38),

ou de celle de son mari :

Edgard eut un bel enterrement, une cérémonie religieuse à l'église de Gentilly. Je le suivis jusqu'au cimetière comme une automate. Ce fut très dur. Il fallait vivre dans ce monde vide, vide sa place à table, vide sa place dans un grand lit où nous avions dormi pendant trente ans. Il ne serait plus là pour Noël, à Pâques, aux anniversaires... Il fallait vivre dignement, sans faiblesse et accomplir et réussir seule la tâche commencée à deux. (Ibid. : 221)

La vie de Madeleine est faite de ruptures, de pertes et de solitude. Dans la vieillesse, elle continue à vivre avec ses chers disparus, sa sœur Jeanne, sa mère et le bien-aimé Edgard :

Même absent, il était là dans la maison qui nous était chère et où nous avions connu des jours heureux. Objets inanimés avez-vous donc une âme? J'étais heureuse parmi tous ces souvenirs. Il était encore là avec moi. (Ibid. : 253)

L'écriture est en ce sens un acte libératoire. L'auteur-narrateur essaie de s'engager dans un travail d'exploration et de compréhension. Elle s'observe, prend de la distance, explique, s'explique. Elle avoue ses doutes, montre ses motivations, ses faiblesses pour justifier parfois sa conduite et affirmer sa vision de la société et de l'existence.

Pourtant, il n'est pas aisé de saisir sa personnalité, car une certaine subjectivité s'inscrit dans son discours marqué aussi inconsciemment par le contexte de l'époque et surtout par le cadre social dans lequel elle évolue. Janet Paterson explique à ce propos que :

Les notions de biographie et d'autobiographie dévoilent leur grande complexité, tout particulièrement en ce qui concerne leur inscription textuelle. Le je auto ou bio, ce je en même temps fictif, ce je qui est une construction discursive par excellence, est de plus en plus difficile à cerner dans son rapport, d'une part, à la tradition littéraire et d'autre part, à ce qu'on appelle le "réel". (Paterson, $2007: 267$ )

L'auteur-narrateur est en effet sensible d'une part au rang social et d'autre part à la couleur de la peau, à la texture des cheveux des personnages; des critères en application dans la société coloniale et encore dans la Guyane du début du siècle.

En ce sens, La Vie d'une Mulâtresse de Cayenne regorge de personnages nantis qui relèvent du même milieu qu'elle: médecins, avocats, sous-directeurs de banques, hommes d'affaires, hauts fonctionnaires. Les beaux Mulâtres « aux yeux verts et coquins ", " aux cheveux fins et légers » (Tichette, $2007: 27$ ), élégants et riches, apparaissent plus souvent 
que les Noirs, tel « le brave gardien martiniquais Carola avec sa belle figure noire éclairée par des yeux intelligents et bons » (Ibid. : 187). Il en est de même des Négresses comme "Camomille, vendeuse au marché, noire comme un pruneau », qui ne paient pas de mine à côté des belles Mulâtresses « claires de peaux avec de jolis cheveux » (Ibid. : 178).

Enfin, l'angoisse intérieure de Madeleine est perceptible dans l'attachement porté au pays natal qu'elle a quitté en 1950. La Guyane joue alors le rôle d'écran sur lequel sont projetées des images discontinues du passé, pour l'exilée souffrant du mal du pays :

Mon pays était loin ; ma maison n'était plus qu'un souvenir, mais j'avais mon mari, mes enfants et je voulais être heureuse. Ma rue Lalouette était toujours dans ma tête, mes amies à des milliers de kilomètres. Il en résulta une dépression nerveuse, mais je réagis pour mes enfants et mon mari qui avaient besoin de mes forces. Peu à peu je me résignai et devins forte et active. (Ibid. : 210)

On s'attendrait, néanmoins, dans La Vie d'une Mulâtresse de Cayenne, à une exploration plus poussée de Paris où elle a vécu plus d'une trentaine d'années. Or, il n'en est rien. La narratrice-sujet ne s'attarde pas sur la capitale. Elle évoque rapidement ses visites au musée Grévin, dans les églises, les cimetières - notamment le Père-Lachaise -, dans les grands magasins comme la Samaritaine, la gentillesse des employés de la mairie de Gentilly où elle réside ou les beaux parterres de roses qui ornent cette ville. C'est qu'elle est hantée par le passé, lorsqu'elle est en France. Elle vit dans un entre-deux temporel et spatial. Et plus précisément, pour reprendre l'expression d'Éric Landowski, on peut dire qu'elle évolue "dans un no man's land, où son identité est sans cesse disloquée, décentrée, fragmentée, brisée » (Landowski, 1997 : 89). Aussi, va-t-elle mettre en place des stratégies qui lui permettront d'être toujours en contact avec la Guyane, sa chère terre natale. La langue créole demeure la langue de communication avec sa vieille servante, complice et amie, Antonine, qui a effectué le déplacement avec la famille et loge chez eux. La vue, l'odorat, le goût à travers les saveurs culinaires permettent à sa mémoire de voyager jusqu'en Guyane :

Il me restait Antonine et notre soixantaine d'années de vie commune. C'était le dernier lien avec mon pays ; nous avons toujours parlé notre vieux créole guyanais, évoqué ensemble nos souvenirs bons et mauvais, parlant des personnes de chez nous. Bons, mauvais, tout y passait. Nous étions toujours fidèles à notre cuisine : haricots, pimentades [...]. Quand les colis arrivaient, il y avait l'odeur du pays, surtout quand ils contenaient de la vanille et de la cannelle... (Tichette, $2007: 226$ )

Néanmoins, Madeleine reste une femme résolument moderne, avec un propos assez audacieux pour l'époque. Son ouvrage est marqué par une certaine tonalité satirique et son humour est parfois décapant. En effet, elle dévoile les manœuvres politiques, révèle les secrets des grandes familles, porte au grand jour les sujets sensibles et tabous, tels que le rapport à la mort ou les relations adultérines :

Le docteur Philippe Pain [...]. Mon parrain [...] s'était marié à Cécile Ursleur, sœur du député, une personne froide qui n'attirait pas. Ils n'avaient pas d'enfant, mais en élevaient deux d'un fils de mon parrain... J'étais au courant de sa vie privée: pendant trente ans ou quarante ans il avait eu une maîtresse et j'étais très émue de les voir l'un près de l'autre dans sa maison du Fort Cépérou, toujours heureux de se retrouver [...]. Cette maîtresse était Inès Florentine, la demi-sœur de maman. (Ibid. : 52)

Elle dénonce comme son prédécesseur Alfred Parépou, dans Atipa, l'hypocrisie religieuse en s'appuyant sur l'éducation élitiste dispensée par les écoles privées tenues par les Sœurs, ou encore sur la « bêtise » de l'Église face aux divorcés qui voudraient se remarier. Elle n'hésite pas à dresser un réquisitoire contre l'administration coloniale en blâmant la 
mesquinerie, voire le racisme de certains fonctionnaires métropolitains affectés en Guyane. Son franc-parler peut déplaire et déranger, mais aussi plaire, d'autant que certaines révélations touchent de près la vie intime des familles de certaines personnalités guyanaises, hommes ou femmes occupant actuellement des postes politiques ou administratifs importants.

La Vie d'une Mulâtresse de Cayenne révèle également la grande culture de Madeleine Tichette, marquée par l'école française. En témoignent non seulement la présence de chansonnettes, de comptines apprises dans les cours de récréation, mais aussi les nombreuses références littéraires disséminées çà et là dans le texte et servant à étayer une démonstration ou à illustrer un comportement. Ainsi, ces paroles chantées dans les défilés carnavalesques et connues d'un grand nombre de Créoles guyanais, «Vaval pou ki sa ou ka léssé nou. Nou pa fait ou arien. » [Vaval pourquoi nous abandonnes-tu? Nous ne t'avons fait aucun mal] (Ibid. : 122), confèrent une certaine authenticité aux faits et gestes des personnages mis en scène, lorsque la période du carnaval est évoquée dans le texte. La valeur véridique du récit ne peut alors être contestée. Il en est de même de ces vers extraits du poème "Ce siècle avait deux ans ", publié par Victor Hugo dans Les Feuilles d'automne, que Tichette cite de mémoire ${ }^{1}$. Ils prolongent le débat engagé par l'auteurnarrateur sur la relation mère-fille et mettent en évidence un amour filial profond:

ô l'amour d'une mère,

Amour que nul n'oublie

Pain merveilleux qu'un dieu partage

Et multiplie

Chacun en a sa part

Et tous l'ont tout entier.

(Ibid. : 41)

Quoi qu'il en soit, toutes ces références extérieures ont le mérite d'apporter un certain dynamisme au récit et de le sauver d'une monotonie qui aurait pu l'enliser.

Dans le cadre d'une invitation lancée par l'une de ses filles installée en Guyane, Madeleine Tichette est revenue en 1980 dans son pays, après une absence de trente ans, pour un séjour qui dura un an. Ce fut son dernier voyage. Elle a prouvé dans les dernières pages de ses Cahiers qu'il peut y avoir continuité entre le passé et le présent. Le «je » raconte un «Moi » avec une distance dans le temps et dans l'espace. Mais le travail de la mémoire peut nier, voire abolir ces barrières :

Aujourd'hui, j'ai quatre-vingt-dix ans. Antonine est toujours là, vieille mais courageuse, me soignant depuis soixante-neuf ans [...]. Je vis depuis 1950 en France, ce pays que j'aime avec son histoire, ses grands hommes, ses monuments, ses belles églises. J'aime ma Guyane où j'ai laissé un peu de mon cœur.

Souvent j'y retourne en pensée et quelquefois en rêve : Un petit tour au cimetière et mon 59 , rue Lalouette où je suis née ainsi que mes enfants. (Ibid. : 280)

La Vie d'une Mulâtresse de Cayenne met en évidence, à travers un dialogue interculturel et intergénérationnel, les problèmes identitaires et existentiels de la Guyane d'hier et d'aujourd'hui. Plus qu'une simple monographie, cet ouvrage constitue aussi un support pour la mémoire collective des habitants de Cayenne. Il met en lumière « l'importance de la mémoire collective dans l'affirmation d'une identité locale et dans l'élaboration d'un imaginaire de référence » (Girard-Hainz, $2005:$ 13). Il prétend exprimer la réalité et manifester une volonté de vérité. Par ailleurs, ce récit de vie a permis à Madeleine Tichette d'évoquer ce qui a été important dans sa vie et de le transmettre à autrui. L'émotion qui se dégage à la lecture de cette œuvre ne tient pas seulement au contenu du 
récit de l'auteur-narrateur. Elle tient aussi au respect que manifestent Francine Château et ses sœurs pour les propos tenus par l'auteur qui est leur mère. Tout le « discours d'escorte ", qui accompagne le lecteur, avant et après le texte narratif, le démontre. Enfin, Fauteur-narrateur entretient avec le lecteur familier de la culture créole guyanaise une relation particulière. En effet, l'évocation des souvenirs de Madeleine Tichette peut entraîner chez celui-ci l'évocation de ses propres souvenirs. Écrire sa vie, c'est essayer de saisir sa personnalité dans sa totalité. C'est tenter de faire une synthèse de soi. En se racontant donc, Madeleine a permis aux autres de la regarder, mais, elle aussi, elle a pu se regarder.

\section{BIBLIOGRAPHIE}

\section{Récits de vie}

Lam Chan, Roger, 1998, De la Guyane au Rhin et Danube, Cayenne, Ibis Rouge Éditions.

Roblin, Yvette, 2004, Guyane je t'aime (malgré tout), Cayenne, Ibis Rouge Éditions.

Tichette, Madeleine, 2007, La Vie d'une Mulâtresse de Cayenne. 1901-1997. Les Cahiers de Madeleine, Paris, L'Harmattan.

Vernet, Claude, 2002, Un enfant de France, Cayenne, RGI.

Vévé, Man, 1994, Man Vévé raconte, Mana, Mairie de Mana.

\section{Autres références}

Abastado, Claude, 1983, « "Raconte ! raconte...". Les récits de vie comme objet sémiotique », Revue des sciences humaines, $\mathrm{n}^{\circ} 191$.

Bertaux, Daniel, 2005, Le Récit de vie. L'enquête et ses méthodes, Paris, Armand Colin.

Candau, Joël, 1998, Mémoire et identité, Paris, PUF.

Cherubini, Bernard, 2002, Interculturalité et créolisation en Guyane française, Paris, L'Harmattan.

Épailly, Eugène, 1995, Une page d'histoire du bagne de Guyane, Alfred Dreyfus déporté dans l'enfer du diable, Kourou, CNES.

Girard-Hainz, Brigitte, 2005, Rêves de ville. Récits d'une vie associative de quartier, Paris, L'Harmattan.

Landowski, Éric, 1997, Présence de l'autre : essais de socio-sémiotique II, Paris, PUF.

Lejeune, Philippe, 1996 [1975], Le Pacte autobiographique, Paris, Seuil, coll. « Points ».

Mam Lam Fouck, Serge, 1996, Histoire générale de la Guyane française des débuts de la colonisation à l'aube de l'an 2000. Les grands problèmes guyanais : permanence et évolution, Cayenne, Ibis Rouge Éditions. 
Parepou, Alfred, 1980, Atipa. Premier roman en créole. 1885, Paris, Éditions Caribéennes.

Paterson, Janet M., 2007, « Biofictions et identités dans L'Immense fatigue des pierres », dans Robert Dion, Frances Fortier, Barbara Havercroft et Hans-Jürgen Lüsebrink (dir.), Vies en récit. Formes littéraires et médiatiques de la biographie et de l'autobiographie, Québec, Éditions Nota bene.

\section{NOTES}

1. Madeleine Tichette cite de manière inexacte le poème de Victor Hugo (voir Victor Hugo, « $\mathrm{Ce}$ siècle avait deux ans", Les Feuilles d'automne [1831], repris dans Euvres poétiques 1, préface par Gaëtan Picon, éd. établie et annotée par Pierre Albouy, Paris, Gallimard, coll. « Bibliothèque de la Pléiade ", 1964, p. 717).

\section{RÉSUMÉS}

Madeleine Tichette est née le 11 novembre 1901 à Cayenne, en Guinée Française. En recherchant ses origines, elle a découvert que son arrière-grand-mère était une jeune négresse. Elle avait été achetée un jour à une vente d'esclaves sur la Place du Marché de Rémire. Quand Madeleine était encore adolescente, elle a perdu sa mère Anna mais a trouvé une seconde mère en sa marraine. Elle est allée à l'école chez les bonnes sœurs dans un établissement nommé Saint-Joseph de Cluny où elle a passé son «certificat d'études ». En 1989, elle a écrit l'histoire de sa vie puis elle est morte alors qu'elle passait ses vacances de Noël à Paris en 1997. Dans cette histoire, nous essaierons d'abord d'analyser le sens donné à plusieurs événements historiques (la période coloniale, les années d'esclavage, La Première Guerre Mondiale et la Seconde Guerre Mondiale, la colonie pénitentiaire...); ensuite, nous nous concentrerons sur la dimension émotionnelle montrée dans le texte par les stratégies narratives utilisées par l'auteur, ainsi que sa relation avec l'imaginaire et l'identité créoles.

Madeleine Tichette was born on 11 November 1901 in Cayenne, in French Guiana. In search of her origins, she found that her great grandmother was a young Negress. She had been bought one day at a slaves' sale, in Rémire's Market Square. When Madeleine was still a teenager, she lost her mother Anna. But she found a second mother in her godmother. She went to a Nuns' school called Saint Joseph of Cluny. There, she received a "school certificate". In 1989, she wrote her life story and died while spending her Christmas holiday in Paris in 1997. In this story, we will first try to analyze the meaning given to several historical events (the colonial period, the slavery days, World Wars I and II, the penal colony...); then we will focus on the emotional dimension shown in the text through the writing strategies used by the author, and its relation to Creole imagination and identity.

\section{INDEX}

Mots-clés : récit de vie, identité, créole, mulâtresse, Guyane

Keywords : life story, identity, mulatoo woman, French Guiana 


\section{AUTEUR}

\section{MONIQUE BLERALD}

Université des Antilles et de la Guyane 